\title{
Neutron Parton Structure And The Light-Front Spectral Function Of 3He
}

\author{
Emanuele Pace* \\ Universita' degli Studi di Roma Tor Vergata, Dipartimento di Fisica, and INFN, Sezione di \\ Roma Tor Vergata, Italy, Via della Ricerca Scientifica 1, 00133 Rome, Italy, \\ E-mail: pace@roma2.infn.it
}

Alessio Del Dotto

Istituto Nazionale di Fisica Nucleare, Sezione di Roma, P.le A. Moro 2, 00185 Rome, Italy and University of South Carolina, Columbia, SC 29208, USA

\section{Leonid Kaptari}

Bogoliubov Lab. Theor. Phys., 141980, JINR, Dubna, Russia

\section{Matteo Rinaldi}

Instituto de Fisica Corpuscular (CSIC-Universitat de Valencia), Parc Cientific UV, C/

Catedratico Jose Beltran 2, E-46980 Paterna (Valencia), Spain

\section{Giovanni Salmè}

Istituto Nazionale di Fisica Nucleare, Sezione di Roma, P.le A. Moro 2, 00185 Rome, Italy

\section{Sergio Scopetta}

Dipartimento di Fisica e Geologia, Università degli Studi di Perugia and INFN, Sezione di Perugia, Via Alessandro Pascoli, 06123 Perugia, Italy

\begin{abstract}
Semi-inclusive deep inelastic electron scattering off polarized ${ }^{3} \mathrm{He}$ is studied in a non-relativistic framework, using a distorted spin-dependent spectral function for ${ }^{3} \mathrm{He}$ to take care of the final state interaction between the observed pion and the remnant. A simple procedure is shown to be valuable for the extraction of Sivers and Collins asymmetries for the neutron from the corresponding asymmetries for ${ }^{3} \mathrm{He}$. To extend this study in a relativistic framework, a novel approach for a Poincaré covariant description of nuclear dynamics is presented, based on the light-front Hamiltonian dynamics. The key quantity is the light-front spectral function, where both normalization and momentum sum rule can be satisfied at the same time. Preliminary results are discussed for an initial analysis of the role of relativity in the EMC effect in ${ }^{3} \mathrm{He}$, and the generalization of the procedure for the extraction of neutron asymmetries within the light-front dynamics is outlined.
\end{abstract}

The 26th International Nuclear Physics Conference

11-16 September, 2016

Adelaide, Australia

* Speaker. 


\section{Introduction}

Semi-inclusive deep inelastic (SIDIS) processes where a fast meson is detected are an important tool for the knowledge of the internal hadron dynamics. Indeed, the detected meson likely originates from the fragmentation of the quark which absorbed the virtual photon and opens a valuable window on the motion of quarks inside the parent nucleon, before the interaction with the photon. Hence, through SIDIS reactions (see, e.g., $[1,2,3]$ ) one can access the transverse-momentumdependent parton distributions (TMDs) of nucleons (see, e.g., Ref. [4]). Neutron targets are not available, but, within a non-relativistic approach which include the final state interaction (FSI) through a distorted spin-dependent spectral function (SF), it has been shown the actual possibility to get information on the neutron structure from SIDIS experiments on ${ }^{3} \mathrm{He}[5,6,7]$.

For a relativistic description of few-body nuclei, we adopt a Poincaré covariant spin-dependent SF [8], built up within the light-front Hamiltonian dynamics (LFHD) for an interacting system with a fixed number of on-mass-shell constituents (see, e.g., [9]). The LFHD has a subgroup structure of the light-front (LF) boosts (with a separation of the intrinsic motion from the global one) and allows one to give a fully Poincaré covariant description of deep inelastic scattering (DIS), SIDIS and deeply virtual Compton scattering. Furthermore, within the LFHD and using the BakamijanThomas (BT) construction of the Poincaré generators [10] one can take advantage of the whole successful non-relativistic (NR) phenomenology that has been developed for the nuclear interaction. A distinct feature of our approach is the ability to implement macrocausality or cluster separability, namely the expected property that if a system is separated into disjoint subsystems by a sufficiently large spacelike separation, then the subsystems behave as independent systems.

In Section 2 the procedure to obtain information on the neutron Collins and Sivers asymmetries from SIDIS experiments on ${ }^{3} \mathrm{He}$ is discussed. In Section 3 the LF spin-dependent (SD) SF obtained from the LF wave functions for two- and three-nucleon systems is described, and the generalization to the LF dynamics of our procedure for the extraction of neutron asymmetries is outlined. In Section 4 the LF SF is applied to study the role of relativity for the EMC effect in ${ }^{3} \mathrm{He}$ and preliminary results are presented. In Section 5 conclusions and perspectives are drawn.

\section{Extraction of neutron asymmetries from SIDIS experiments off ${ }^{3} \mathrm{He}$}

The Collins and Sivers asymmetries, $A_{3}^{C(S)}$, can be expressed as follows

$$
A_{3}^{C(S)}=\frac{\int_{x}^{A} d \alpha\left[\Delta \sigma_{C(S)}^{n}\left(x / \alpha, Q^{2}\right) f_{n}^{\perp}\left(\alpha, Q^{2}\right)+2 \Delta \sigma_{C(S)}^{p}\left(x / \alpha, Q^{2}\right) f_{p}^{\perp}\left(\alpha, Q^{2}\right)\right]}{\int d \alpha\left[\sigma^{n}\left(x / \alpha, Q^{2}\right) f_{n}\left(\alpha, Q^{2}\right)+2 \sigma^{p}\left(x / \alpha, Q^{2}\right) f_{p}\left(\alpha, Q^{2}\right)\right]}
$$

in terms of the light-cone unpolarized, $f_{N}$, and transverse, $f_{N}^{\perp}$, momentum distributions (md)

$$
f_{N}^{(\perp)}\left(\alpha, Q^{2}\right)=\int d E \int_{p_{m}\left(\alpha, Q^{2}\right)}^{p_{M}\left(\alpha, Q^{2}\right)} \frac{m_{N}}{E_{N}} P_{N}^{(\perp)}(E, \mathbf{p}) \delta\left(\alpha-\frac{p \cdot q}{m_{N} v}\right) \theta\left(W_{Y}^{2}-\left(m_{N}+m_{\pi}\right)^{2}\right) d^{3} \mathbf{p}
$$

with $W_{Y}$ the invariant mass of the debris $\mathrm{Y}$, which hadronizes in a nucleon and, at least, one pseudoscalar meson. The quantities $\Delta \sigma_{C(S)}^{N}$ and $\sigma^{N}$ in Eq. (2.1) are related to the structure of the bound nucleon

$$
\Delta \sigma_{C}^{N}\left(x, Q^{2}\right)=\frac{1-y}{1-y-y^{2} / 2}
$$




$$
\begin{array}{r}
\times \sum_{q} e_{q}^{2} \int d^{2} \kappa_{T} d^{2} \mathbf{k}_{T} \delta^{2}\left(\mathbf{k}_{T}+\mathbf{q}_{T}-\kappa_{T}\right) \frac{\hat{\mathbf{P}}_{h \perp} \cdot \kappa_{T}}{m_{h}} h_{1}^{q, N}\left(x, \mathbf{k}_{T}^{2}\right) H_{1}^{\perp q, h}\left(z,\left(z \kappa_{T}\right)^{2}\right) \\
\Delta \sigma_{S}^{N}\left(x, Q^{2}\right)=\sum_{q} e_{q}^{2} \int d^{2} \kappa_{T} d^{2} \mathbf{k}_{T} \delta^{2}\left(\mathbf{k}_{T}+\mathbf{q}_{T}-\kappa_{T}\right) \frac{\hat{\mathbf{P}}_{h \perp} \cdot \mathbf{k}_{\mathbf{T}}}{m_{N}} f_{1 T}^{\perp q, N}\left(x, \mathbf{k}_{T}^{2}\right) D_{1}^{q, h}\left(z,\left(z \kappa_{T}\right)^{2}\right),(2.4 \\
\sigma^{N}\left(x, Q^{2}, z\right)=\sum_{q} e_{q}^{2} \int d^{2} \kappa_{T} d^{2} \mathbf{k}_{T} \delta^{2}\left(\mathbf{k}_{T}+\mathbf{q}_{T}-\kappa_{T}\right) f_{1}^{q, N}\left(x, \mathbf{k}_{T}^{2}\right) D_{1}^{q, h}\left(z,\left(z \kappa_{T}\right)^{2}\right)
\end{array}
$$

where $z=E_{h} / v$ and models for the parton distributions $h_{1}^{q, N}, f_{1 T}^{\perp q, N}, f_{1}^{q, N}$, and for the fragmentation functions $H_{1}^{\perp q, h}, D_{1}^{q, h}$ were used (see Ref. [11]). In Eq. (2.2) $P_{N}(E, \mathbf{p})$ is the unpolarized SF (see [12]), while $P_{N}^{\perp}(E, \mathbf{p})$ is the transverse $\mathrm{SF}$

$$
P_{N}^{\perp}(E, \mathbf{p})=\Re e\left\{P_{\frac{1}{2}-\frac{1}{2}}^{N \frac{1}{2}-\frac{1}{2}}(E, \mathbf{p})+P_{\frac{1}{2}-\frac{1}{2}}^{N-\frac{1}{2} \frac{1}{2}}(E, \mathbf{p})\right\} .
$$

In Ref. [7] the matrix elements of a distorted SD SF which includes a generalized eikonal approximation (GEA) to take care of the FSI in a NR approach were introduced

$$
P_{\lambda \lambda^{\prime}}^{N M M^{\prime}}(E, \mathbf{p})=\sum_{f_{23}} \sum_{\varepsilon_{23}^{*}} \rho\left(\varepsilon_{23}^{*}\right) \tilde{\mathscr{O}}_{\lambda \lambda^{\prime}}^{N M M^{\prime} f_{23}}\left(\varepsilon_{23}^{*}, \mathbf{p}\right) \delta\left(E+M_{3}-m_{N}-M_{23}^{*}\right)
$$

with $\quad \tilde{\mathscr{O}}_{\lambda \lambda^{\prime}}^{N M M^{\prime} f_{23}}\left(\varepsilon_{23}^{*}, \mathbf{p}\right)=\left\langle\lambda, \mathbf{p} ; \hat{S}_{G l} \phi_{\varepsilon_{23}^{*}}^{f_{23}} \mid \Psi_{3}^{M}\right\rangle\left\langle\Psi_{3}^{M^{\prime}} \mid \lambda^{\prime}, \mathbf{p} ; \hat{S}_{G l} \phi_{\varepsilon_{23}^{*}}^{f_{23}}\right\rangle$.

The spin components $M, M^{\prime}$ and $\lambda, \lambda^{\prime}$ are defined with respect to the direction of $\hat{\mathbf{q}}$. The operator $\hat{S}_{G l}\left(\mathbf{r}_{1}, \mathbf{r}_{2}, \mathbf{r}_{3}\right)=\prod_{i=2,3}\left[1-\theta\left(z_{i}-z_{1}\right) \Gamma\left(\mathbf{b}_{1}-\mathbf{b}_{i}, z_{1}-z_{i}\right)\right]$ is a Glauber operator which takes care of hadronization and FSI. The model of Ref. [13] for the (generalized) profile function $\Gamma(\mathbf{b}, z)$, already successfully applied to ${ }^{2} H\left(e, e^{\prime} p\right) X[14]$, is adopted.

In Ref. [11], using the NR SF of Ref. [12] and within the plane wave impulse approximation (IA), i.e. no interaction between the measured fast $\pi$, the remnant debris and the interacting twonucleon recoiling system, it was shown that the formula [15]

$$
A_{n} \simeq \frac{1}{p_{n} d_{n}}\left(A_{3}^{e x p}-2 p_{p} d_{p} A_{p}^{e x p}\right)
$$

already widely used to extract neutron asymmetries in DIS from experiments on ${ }^{3} \mathrm{He}$, works also in SIDIS, both for the Collins and Sivers single spin asymmetries. Nuclear effects are hidden in the effective polarizations (EP) $p_{p}=-0.024$ and $p_{n}=0.878$ and in the dilution factors, $d_{p(n)}$.

To investigate whether the formula (2.8) can be safely applied even in presence of the FSI, the GEA distorted spin-dependent SF was adopted in Ref. [7]. While $P^{I A}$ depends on ground state properties, $P^{F S I}$ is process dependent, since the Glauber operator depend on the kinematics of the process. Then for each experimental point $\left(x, Q^{2} \ldots\right)$ a different $P^{F S I}$ has to be evaluated !

The SFs $P^{I A}$ and $P^{F S I}$, as well as the light-cone md $f_{N}^{I A}$ and $f_{N}^{F S I}$ can be very different (see Fig. 1) and therefore FSI's have a strong effect on the SIDIS cross sections. However, including the FSI the md $f_{N}$ and $f_{N}^{\perp}$ change in the same way and in asymmetries the md appear both in the numerator and in the denominator. Furthermore, while FSI's change effective polarizations $p_{p(n)}$ by $10-15 \%$, it occurs that effects of GEA-FSI in the dilution factors and in the EP compensate each other to a large extent: i.e., the products $p_{p(n)}^{F S I} d_{p(n)}^{F S I}$ and $p_{p(n)}^{I A} d_{p(n)}^{I A}$ are essentially the same [6]. Then the usual extraction of Eq. (2.8) is safe, as shown at $\mathscr{E}=8.8 \mathrm{GeV}$ in Fig. 2. 

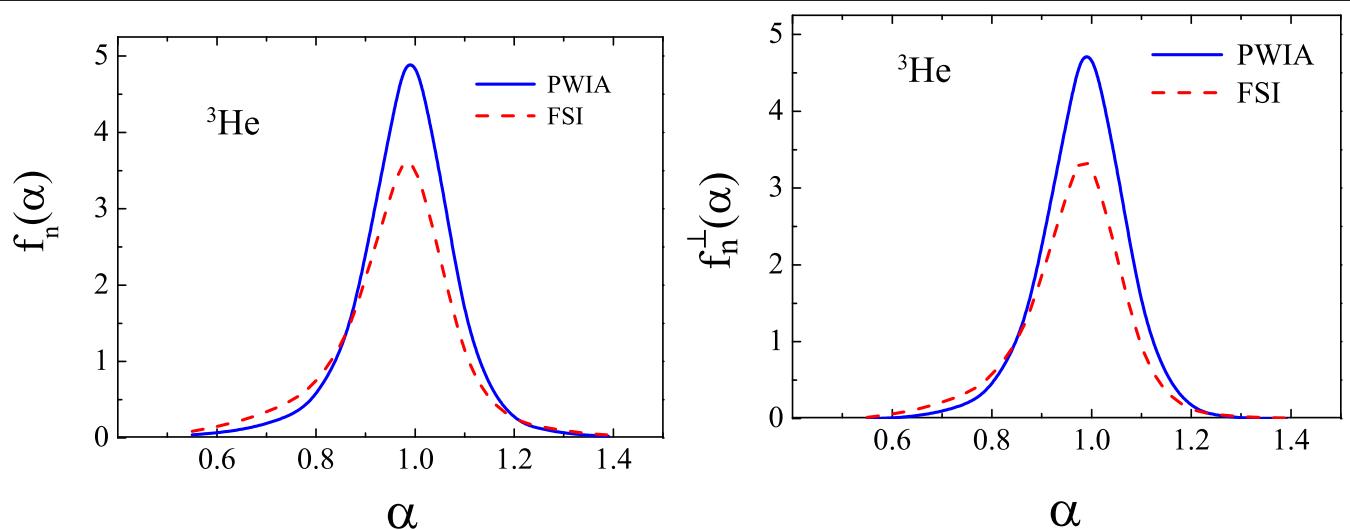

Figure 1: Neutron unpolarized and transversely polarized distributions in ${ }^{3} \mathrm{He}$ in IA (full lines) and with FSI (dashed lines) for the initial electron energy $\mathscr{E}=8.8 \mathrm{GeV}$ and $Q^{2}=5.73(\mathrm{GeV} / \mathrm{c})^{2}$ (preliminary results).
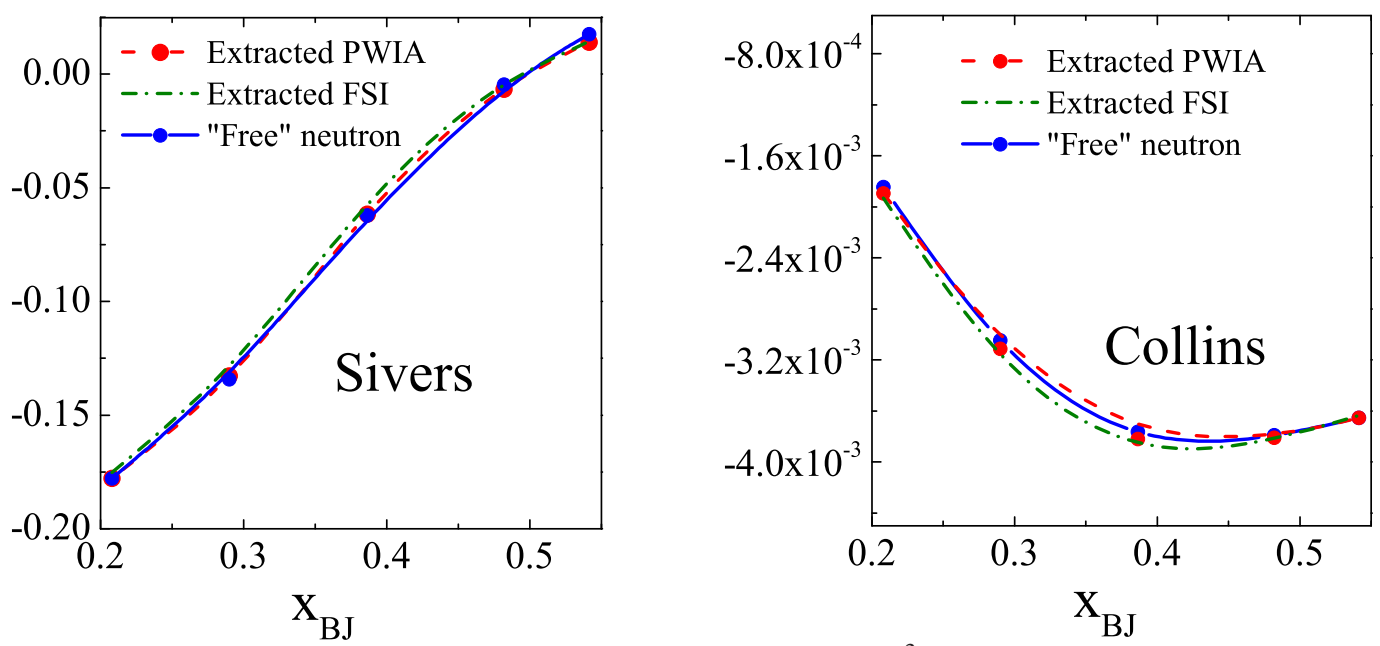

Figure 2: Neutron asymmetries extracted (Eq. (2.7)) from the ${ }^{3} \mathrm{He}$ Sivers (left panel) and Collins (right panel) asymmetries, with and without FSI, in the actual kinematics of JLab [2] (preliminary results, see [7]).

\section{Light-Front Dynamics and the Light-Front Spectral Function}

An explicit construction of the 10 Poincare generators that fulfills the proper commutation rules in presence of interactions was given by Bakamjian and Thomas [10] : i) only the mass operator, $\mathrm{M}$, contains the interaction, and ii) it generates the dependence upon the interaction of the three dynamical generators in LFHD, namely $P^{-}$and the transverse rotations $\vec{F}_{\perp}$. M is obtained adding to the free mass $M_{0}$ of the system an interaction $V$. There are two possibilities: $M^{2}=$ $M_{0}^{2}+U$ (then for two particles one can easily embed the NR phenomenology) or $M=M_{0}+V$. The interaction, $U$ or $V$, must commute with all the kinematical generators, and with the non interacting spin. Then it has to be invariant for translations and rotations, as in the NR case.

For the three-body case the mass operator is $M_{B T}(123)=M_{0}(123)+V^{B T}$, where $M_{0}(123)=$ $\sqrt{m^{2}+k_{1}^{2}}+\sqrt{m^{2}+k_{2}^{2}}+\sqrt{m^{2}+k_{3}^{2}}$ is the free mass operator, $V^{B T}$ a BT two-body and three-body force, and $k_{i}(i=1,2,3)$ are intrinsic momenta with $\mathbf{k}_{1}+\mathbf{k}_{2}+\mathbf{k}_{3}=0$ [9].

The NR mass operator is written as $M^{N R}=3 m+\sum_{i=1,3} k_{i}^{2} / 2 m+V_{12}^{N R}+V_{23}^{N R}+V_{31}^{N R}+V_{123}^{N R}$ and must obey the commutation rules proper of the Galilean group, leading to translational and rotational invariance. Those properties are analogous to the ones in the BT construction. This 
allows us to consider the standard NR mass operator as a sensible BT mass operator, and embed it in a Poincaré covariant approach: $M_{B T}(123) \sim M^{N R}$.

To obtain within the LFHD a Poincaré-covariant spin-dependent SF for a three-particle system in the bound state $\left|\Psi_{0} ; S, T_{z}\right\rangle$, eigenstate of the mass operator $M_{B T}(123)$ and polarized along $\vec{S}$, let us use the LF overlaps ${ }_{L F}\left\langle\tau_{S}, T_{S} ; \alpha, \varepsilon ; J_{z} J ; \tau \sigma, \tilde{\kappa} \mid \Psi_{0} ; S, T_{z}\right\rangle$ in place of their NR counterparts in the definition of the SF. The state ${ }_{L F}\left\langle\tau_{S}, T_{S} ; \alpha, \varepsilon ; J_{z} J ; \tau \sigma, \tilde{\kappa}\right|$ is the tensor product of a plane wave for the knocked-out constituent (say particle 1 ) with intrinsic momentum $\tilde{\kappa}$, and a fully interacting intrinsic state for the spectator system (say particles 2 and 3), with energy $\varepsilon$, all moving in the intrinsic reference frame of the cluster $(1,23)$. When applications to DIS or SIDIS processes are concerned, the issue of macrocausality has to be considered, i.e., if the subsystems which compose a system are brought far apart, the Poincaré generators of the system have to become the sum of the Poincaré generators corresponding to the subsystems in which the system is asymptotically separated. The packing operators [9], which make it possible to include the macrocausality in the bound state, are not considered in the present approximation. However, we implement macrocausality in the tensor product of a plane wave for the knocked-out constituent times a fully interacting intrinsic state for the spectator pair. Then, the LF spin-dependent SF for the three-nucleon system $\left({ }^{3} \mathrm{He}\right.$ or $\left.{ }^{3} \mathrm{H}\right)$ is [8]

$$
\begin{aligned}
& \mathscr{P}_{\sigma^{\prime} \sigma}^{\tau}\left(\xi, \kappa_{\perp}, \kappa^{-}, S\right)=\left|\frac{\partial \kappa^{+}}{\partial \xi}\right| \sum_{d} d \varepsilon \rho(\varepsilon) \delta\left(\kappa^{-}-M_{3}+\frac{M_{S}^{2}+\left|\kappa_{\perp}\right|^{2}}{(1-\xi) M_{3}}\right) \times \\
& \sum_{J J_{z} \alpha} \sum_{T_{S} \tau_{S}}{ }_{L F}\left\langle\tau_{S}, T_{S}, \alpha, \varepsilon ; J J_{z} ; \tau \sigma^{\prime}, \tilde{\kappa} \mid \Psi_{0} ; S, T_{z}\right\rangle\left\langle S, T_{z} ; \Psi_{0} \mid \tilde{\kappa}, \sigma \tau ; J J_{z} ; \varepsilon, \alpha, T_{S}, \tau_{S}\right\rangle_{L F}
\end{aligned}
$$

where $\tau= \pm 1 / 2, M_{3}$ is the nucleus mass, $\rho(\varepsilon)$ the density of the two-nucleon eigenstates $(\rho(\varepsilon)=$ $\sqrt{\varepsilon m} m / 2$ for the two-body continuum states and $\rho(\varepsilon)=1$ for the deuteron bound state), $J$ the spin and $T_{S}$ the isospin of the two-body state, $\alpha$ the set of quantum numbers needed to completely specify this eigenstate, and $M_{S}=2 \sqrt{m^{2}+m \varepsilon}$ its mass. From $\xi, M_{S}, \kappa_{\perp}$ one can define $\kappa^{+}=$ $\xi \mathscr{M}_{0}(1,23)$, where $\mathscr{M}_{0}(1,23)$ is the free mass of the cluster $(1,23)$

$$
\mathscr{M}_{0}^{2}(1,23)=\frac{m^{2}+\left|\kappa_{\perp}\right|^{2}}{\xi}+\frac{M_{S}^{2}+\left|\kappa_{\perp}\right|^{2}}{(1-\xi)} .
$$

The overlap ${ }_{L F}\left\langle\tau_{S}, T_{S} ; \alpha, \varepsilon ; J_{z} J ; \tau \sigma, \tilde{\kappa} \mid \Psi_{0} ; S, T_{z}\right\rangle$ is defined as follows [8]

$$
\begin{aligned}
& L_{F}\left\langle\tau_{S}, T_{S}, \alpha, \varepsilon ; J_{z} J ; \tau \sigma, \tilde{\kappa} \mid \Psi_{0} ; S, T_{z}\right\rangle=\sum_{\tau_{2}, \tau_{3}} \int d \mathbf{k}_{23} \sum_{\sigma_{1}^{\prime}} D^{\frac{1}{2}}\left[\mathscr{R}_{M}(\tilde{k})\right]_{\sigma \sigma_{1}^{\prime}} \sqrt{\frac{\kappa^{+} E_{23}}{k^{+} E_{S}}} \sqrt{(2 \pi)^{3} 2 E(\mathbf{k})} \times \\
& \sum_{\sigma_{2}^{\prime \prime}, \sigma_{3}^{\prime \prime}} \sum_{\sigma_{2}^{\prime}, \sigma_{3}^{\prime}} \sum_{\sigma_{2}} D^{\frac{1}{2}}\left[R_{M}^{\dagger}\left(\tilde{\mathbf{k}}_{23}\right)\right]_{\sigma_{2}^{\prime \prime} \sigma_{2}} D^{\frac{1}{2}}\left[R_{M}\left(\tilde{\mathbf{k}}_{2}\right)\right]_{\sigma_{2} \sigma_{2}^{\prime}} \sum_{\sigma_{3}} D^{\frac{1}{2}}\left[R_{M}^{\dagger}\left(-\tilde{\mathbf{k}}_{23}\right)\right]_{\sigma_{3}^{\prime \prime} \sigma_{3}} D^{\frac{1}{2}}\left[R_{M}\left(\tilde{\mathbf{k}}_{3}\right)\right]_{\sigma_{3} \sigma_{3}^{\prime}} \\
& \quad \times \quad I_{I F}\left\langle\tau_{S}, T_{S}, \alpha, \varepsilon ; J_{z} J \mid \mathbf{k}_{23} ; \sigma_{2}, \sigma_{2}{ }_{3} ; \tau_{2}, \tau_{3}\right\rangle\left\langle\tau_{3}, \tau_{2}, \tau ; \sigma_{3}^{\prime}, \sigma_{2}^{\prime}, \sigma_{1}^{\prime} ; \mathbf{k}, \mathbf{k}_{23} \mid \Psi_{0} ; S, T_{z}\right\rangle_{I F}
\end{aligned}
$$

where $\mathbf{k}_{23}$ is the intrinsic momentum of the (23) pair, $\mathbf{k}$ is the intrinsic nucleon momentum in the (123) system $\left(\mathbf{k}_{\perp}=\kappa_{\perp}\right.$, since we choose the ${ }^{3} \mathrm{He}$ transverse momentum $\left.\mathbf{P}_{\perp}=0\right), k^{+}=$ $\xi M_{0}(123)=\kappa^{+} M_{0}(123) / \mathscr{M}_{0}(1,23)$, with $M_{0}(123)$ the free mass of the three-particle system

$$
M_{0}^{2}(123)=\frac{m^{2}+\left|k_{\perp}\right|^{2}}{\xi}+\frac{M_{23}^{2}+\left|k_{\perp}\right|^{2}}{(1-\xi)}
$$


and $M_{23}^{2}=4\left(m^{2}+\left|\mathbf{k}_{23}\right|^{2}\right)$ the mass of the spectator pair without interaction! In Eq. (3.3) one has $k_{z}=\frac{1}{2}\left[k^{+}-\left(m^{2}+\left|\kappa_{\perp}\right|^{2}\right) / k^{+}\right], E_{23}=\sqrt{M_{23}^{2}+|\mathbf{k}|^{2}}$ and $E_{S}=\sqrt{M_{S}^{2}+|\kappa|^{2}}$. Furthermore $D_{\sigma, \sigma^{\prime}}^{s}\left(R_{M}^{\dagger}(\tilde{\mathbf{k}})\right)$ is the Wigner function, needed for coupling angular momenta in LFHD, and the Melosh rotation $R_{M}(\tilde{\mathbf{k}})$ is the rotation between the rest frames of the particle reached through a LF boost or a canonical, rotationless boost [9]. In our calculations, we identify the instant form (IF) overlaps of Eq. (3.3) with the NR wave functions for the two-nucleon and the three-nucleon [16] systems, corresponding to the NN interaction AV18 [17].

We are presently planning to test our extraction procedure of neutron asymmetries from ${ }^{3} \mathrm{He}$ asymmetries using the LF SF and including in our LF description the FSI between the jet produced from the hadronizing quark and the two-nucleon spectator system through an extension to the LF framework of the GEA of Refs. [13, 14], as we did in the NR case [5, 7].

\section{Light-front momentum distribution and preliminary results for the EMC effect}

From the LF SF one can obtain the momentum distribution $f_{p(n)}^{A}(z)$

$$
f_{\tau}^{A}(z)=\int_{0}^{1} d \xi \int d \kappa_{\perp} \int d \kappa^{-} \frac{1}{2(2 \pi)^{3} \kappa^{+}} \operatorname{Tr}\left[\mathscr{P}^{\tau}\left(\xi, \mathbf{k}_{\perp}, \kappa^{-}, S\right)\right] \delta\left(z-\frac{\xi M_{A}}{m}\right)
$$

that naturally fulfills normalization and momentum sum rule

$$
\int_{0}^{M_{A} / m} d z f_{\tau}^{A}(z)=1 \quad M S R=\frac{1}{A} \int_{0}^{M_{A} / m} d z z\left[Z f_{p}^{A}(z)+(A-Z) f_{n}^{A}(z)\right]=\frac{M_{A}}{A m}
$$

because of the symmetry of the three-body bound state (see [8]). To investigate whether the LF SF can affect the EMC effect, we first evaluated the nuclear structure function $F_{2}^{A}(x)\left(x=Q^{2} / 2 m v\right)$ as a convolution of the nuclear SF and of the nucleon structure function for the proton and the neutron. Then we obtained the ratios

$$
R_{2}^{A}(x)=\frac{F_{2}^{A}(x)}{Z F_{2}^{p}(x)+(A-Z) F_{2}^{n}(x)}
$$

and $R_{2}^{H e}(x) / R_{2}^{D}(x)$. For the two-body channel an exact calculation was performed. In the threebody channel average values for $k_{23}$ were inserted in Eq. (3.3). Our preliminary results are shown in Fig. 3 and encourage us in performing the full LF calculation.

\section{Conclusions and Perspectives}

An investigation of SIDIS processes off ${ }^{3} \mathrm{He}$ beyond the NR, impulse approximation approach is presently being carried out. A Generalized Eikonal Approximation has been used to deal with the FSI effects and a distorted spin-dependent spectral function, still non relativistic, has been defined $[5,7]$. It has been shown that the formula (2.8) can be safely used to obtain both the Collins and Sivers neutron asymmetries from the measured Collins and Sivers asymmetries of ${ }^{3} \mathrm{He}$ [7].

A Poincaré covariant description of $A=3$ nuclei, based on the LFHD, has been proposed [8]. The BT construction of the Poincaré generators allows one to embed the successful NR phenomenology for few-nucleon systems in a Poincaré covariant framework. Then a LF SF can be 


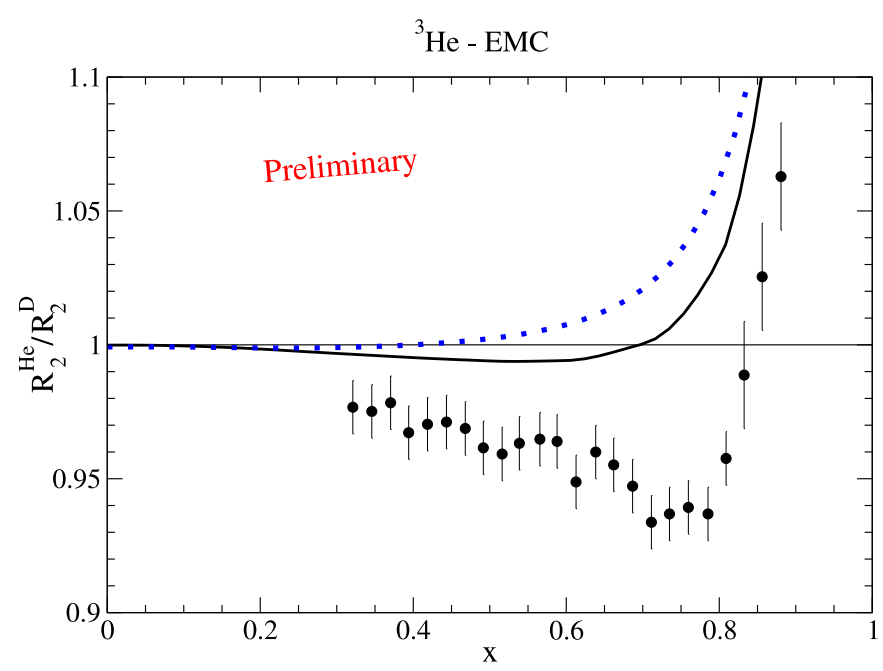

Figure 3: ${ }^{3} \mathrm{He}$ EMC effect. Solid line: result for the LF SF, with exact calculation in the 2-body channel, and average energies in the 3-body one: $\left\langle k_{23}>=113.53 \mathrm{MeV}\right.$ (proton), $<k_{23}>=91.27 \mathrm{MeV}$ (neutron), corresponding to the average kinetic energy of the intrinsic motion of the (23) pair in the continuum spectrum. Dotted line: result with the approach of Ref. [18] for the SF. Experimental data are from Ref. [19].

defined that exactly fulfills both the normalization and the momentum sum rule. The nucleon SF for ${ }^{3} \mathrm{He}$, has been evaluated by approximating the IF overlaps in Eq. (3.3) with their NR counterparts, calculated with the AV18 NN interaction, since it fulfills rotational and translational symmetries.

Let us stress two important features of our LF spectral function : i) the definition of the nucleon momentum $\tilde{\kappa}$ in the intrinsic reference frame of the cluster $(1,23)$; and ii) the use for the calculation of the LF spectral function of the tensor product of a plane wave of momentum $\tilde{\kappa}$ times the state which describes the intrinsic motion of the fully interacting spectator subsystem. These new features allows one to take care of macrocausality and to introduce a new effect of binding in the spectral function.

A first test of our approach is the EMC effect for ${ }^{3} \mathrm{He}$. The 2-body contribution to the nucleon SF has been calculated with the full expression, while for the 3-body contribution average values for $<k_{23}^{2}>$ have been used. In the comparison with experimental data, encouraging improvements clearly appear with respect to the non-relativistic result. Therefore, relativistic effects generated by the fulfillment of Poincaré covariance at the nucleus level, seem to be required to identify unambiguously new, genuine QCD phenomena inside the nucleus itself.

Our next steps will be the full calculation of the EMC effect for ${ }^{3} \mathrm{He}$, including the exact 3-body contribution, and the introduction of the FSI through the GEA within the LFHD.

\section{References}

[1] X. Qian et al. [Jefferson Lab Hall A Collaboration], Single Spin Asymmetries in Charged Pion Production from Semi-Inclusive Deep Inelastic Scattering on a Transversely Polarized ${ }^{3} \mathrm{He}$ Target at $Q^{2}=1.4-2.7 \mathrm{GeV}^{2}$, Phys. Rev. Lett. 107 (2011) 072003.

[2] H. Gao et al., Transverse spin structure of the nucleon through target single-spin asymmetry in semi-inclusive deep-inelastic ( $e, e^{\prime} \pi^{ \pm}$) reaction at Jefferson Lab, Eur. Phys. J. Plus 126 (2011) 2; G. Cates et al., E12-09-018, JLAB approved experiment; J.P. Chen et al., PR12-11-007 (Rating A), 
Asymmetries in Semi-Inclusive Deep-Inelastic ( $\left.e, e^{\prime} \pi^{ \pm}\right)$Reactions on a Longitudinally Polarized ${ }^{3} H e$ Target.

[3] J.-P. Chen, X. Jiang and J.-C. Peng, E-06-010 Proposal to JLab-PAC29, E. Cisbani, H. Gao and X. Jiang, E-06-011 Proposal to JLab-PAC29, Measurement of Single Target-Spin Asymmetry in Semi-Inclusive Pion Electroproduction on a Transversely Polarized ${ }^{3}$ He Target.

[4] V. Barone, F. Bradamante and A. Martin, Transverse-spin and transverse-momentum effects in high-energy processes, Prog. Part. Nucl. Phys. 65 (2010) 267.

[5] L. Kaptari, A. Del Dotto, E. Pace, M. Rinaldi, G. Salmé, S. Scopetta, Distorted spin-dependent spectral function of an $A=3$ nucleus and semi-inclusive deep inelastic scattering processes, Phys. Rev. C 89 (2014) 035206.

[6] A. Del Dotto, L. Kaptari, E. Pace, G. Salmé, S. Scopetta, Towards an Improved Description of SiDIS by a Polarized ${ }^{3}$ He Target, Few Body Syst. 55 (2014) 877-880.

[7] L. Kaptari, A. Del Dotto, E. Pace, G. Salmé, S. Scopetta, to be published.

[8] A. Del Dotto, E. Pace, G. Salmé, S. Scopetta, Light-front spin-dependent spectral function and nucleon momentum distributions for a three-body system, Phys. Rev. C 95 (2017) 014001.

[9] B.D. Keister and W.N. Polyzou, Relativistic Hamiltonian Dynamics in Nuclear and Particle Physics, Adv. Nucl. Phys. 21 (1991) 225-550.

[10] B. Bakamjian and L.H. Thomas, Relativistic Particle Dynamics. II*, Phys. Rev. 92 (1953) 1300-1310.

[11] S. Scopetta, Neutron single spin asymmetries from semi-inclusive deep inelastic scattering off transversely polarized ${ }^{3}$ He. Phys. Rev. D 75 (2007) 054005-1.

[12] C. Ciofi degli Atti, E. Pace, G. Salmè, Spin dependent spectral function of ${ }^{3}$ He and the asymmetry in the process polarized ${ }^{3} \mathrm{He}$ (polarized e, e') X, Phys. Rev. C 46 (1992) R1591; C. Ciofi degli Atti, E. Pace, G. Salmè, Investigation of the neutron form factors by inclusive quasielastic scattering of polarized electrons off polarized ${ }^{3} \mathrm{He}$ : a theoretical overview, Phys. Rev. C 51 (1995) 1108-1119.

[13] C. Ciofi degli Atti, B.Z. Kopeliovich, Final state interaction in semiinclusive DIS off nuclei, Eur. Phys. J. A17 (2003) 133-144.

[14] C. Ciofi degli Atti and L. Kaptari, Semi-inclusive deep-inelastic scattering off few-nucleon systems: Tagging the EMC effect and hadronization mechanisms with detection of slow recoiling nuclei, Phys. Rev. C 83 (2011) 044602-1,044602-8.

[15] C. Ciofi degli Atti, S. Scopetta, E. Pace, G. Salmé, Nuclear effects in deep inelastic scattering of polarized electrons off polarized ${ }^{3} \mathrm{He}$ and the neutron spin structure functions, Phys. Rev. C 48 (1993) R968-R972.

[16] A. Kievsky, M. Viviani, and S. Rosati, Study of bound and scattering states in three-nucleon systems, Nucl. Phys. A 577 (1994) 511-527.

[17] R.B. Wiringa, V.G.J. Stocks, and R. Schiavilla, Accurate nucleon-nucleon potential with charge-independence breaking, Phys. Rev. C 51 (1995) 38-51.

[18] U. Oelfke, P. Sauer and F. Coester, Convolution models of deep inelastic scattering: the three-nucleon bound state as a test case, Nucl. Phys. A 518 (1990) 593-616.

[19] J. Seely et al., New Measurements of the European Muon Collaboration Effect in Very Light Nuclei. Phys. Rev. Lett. 103 (2009) 202301-1;202301-5. 